\title{
Generalised Algebraic Continued Fractions related to Definite Integrals ${ }^{1}$
}

\author{
By L. R. Shenton \\ (Received 11th May, 1955.)
}

\section{Introduction.}

The present paper is a continuation of the work initiated in [1]-[5]. In [5] I gave an expansion of the form

$$
a_{0}\left|V_{s}, W_{s+1}\right| \div\left|U_{s}, V_{s+1}\right|
$$

for the second order C.F. associated with

$$
F\left(z_{1}, z_{2}\right)=\int_{0}^{\infty} \frac{d \psi(x)}{\left(x+z_{1}\right)\left(x+z_{2}\right)}
$$

where $U_{s}, V_{s}, W_{s}$ satisfy a fourth-order recurrence relation, there being a similar expansion for third order C.F.'s. I shall now give simple expressions for $U_{s}, V_{s}, W_{s}$ (or related forms) in terms of $\chi_{2 s}\left(z_{1}\right), \chi_{2 s}\left(z_{2}\right)$, $\omega_{28}\left(z_{1}\right), \omega_{2 s}\left(z_{2}\right)$, where

$$
\frac{\chi_{2 s}(x)}{\omega_{2 s}(x)}=\frac{a_{0}}{x+c_{1}}-\frac{a_{1}}{x+c_{2}}-\ldots \quad \frac{a_{s-1}}{x+c_{s}},
$$

and show that there is a remarkable relation between the recurrence formula for the first order C.F. and that satisfied by $U_{s}, V_{s}, W_{s}$. The generalised form of these results will be stated and proved.

Some remarks on other forms of generalisations of C.F's are given in conclusion, reference being made to the correspondence between Stieltjes and Hermite on this subject.

\section{Expansions involving symmetric functions.}

The basis of the discussion is the function defined by

$$
F(z)=\frac{1}{k_{1} z}+\frac{1}{k_{2}}+\frac{1}{k_{3} z}+\frac{1}{k_{4}}+\ldots
$$

1 By an algebraic continued fraction we mean one whose partial denominators (and perhaps numerators) are functions of a single variable $x$, contrasting with a C.F. with numerical elements. 
in which it is assumed that the $k$ 's are positive and $\Sigma k_{s}$ diverges. This implies the existence of a unique bounded non-decreasing function $\psi(x)$ in the interval $(0, \infty)$ such that

$$
F(z)=\int_{0}^{\infty} \frac{d \psi(x)}{x+z}
$$

For our present purpose a form equivalent to (1) is preferred, namely

$$
F(z)=\frac{b_{1}}{z}+\frac{b_{2}}{1}+\frac{b_{3}}{z}+\frac{b_{4}}{1}+\ldots \quad\left(=\frac{\chi_{s}(z)}{\omega_{s}(z)} \text { as } s \rightarrow \infty\right)
$$

and its "contracted" form 1

$$
F(z)=\frac{a_{0}}{z+c_{1}}-\frac{a_{1}}{z+c_{2}}-\frac{a_{2}}{z+c_{3}}-\ldots \quad\left(=\frac{P_{s}(z)}{Q_{s}(z)} \text { as } s \rightarrow \infty\right) .
$$

Let $A(x), B(x)$ and $C(x)$ be polynomials with real coefficients, $A(x)$ of degree $n$ or less, $B(x)$ of degree less than $n$, and suppose

$$
C(x)=\prod_{\lambda=1}^{n}\left(x+z_{\lambda}\right)>0 \quad \text { for } \quad x \geqslant 0, \quad z_{\lambda} \text { distinct. }
$$

From (28) of $\S 3$ [4], we have an $n$th order C.F. given by

$$
\begin{aligned}
F\left(z_{\lambda}\right\rangle_{1}^{n} & =\int_{0}^{\infty} \frac{A(x) B(x)}{C(x)} d \psi(x) \\
& =\lim _{s \rightarrow \infty} \frac{P_{s}(A, B)}{Q_{s}\left(z_{\lambda}\right)_{1}^{n}},
\end{aligned}
$$

where

$$
\begin{aligned}
Q_{s}\left(z_{\lambda}\right)_{1}^{n} & =\left|Q_{s}\left(z_{1}\right), Q_{s+1}\left(z_{2}\right), \ldots, Q_{s+n-1}\left(z_{n}\right)\right| / \Delta, \\
\Delta & =\left|z_{1}^{0}, z_{2}^{1}, \ldots, z_{n}^{n-1}\right|,
\end{aligned}
$$

$$
P_{s}(A, B)=\frac{(-1)^{n}}{\Delta}\left|\begin{array}{ccccc}
-(A, B) & W_{s}(B) & W_{s+1}(B) & \ldots & W_{s+n-1}(B) \\
A\left(-z_{1}\right) & Q_{s}\left(z_{1}\right) & Q_{s+1}\left(z_{1}\right) & \ldots & Q_{s+n-1}\left(z_{1}\right) \\
\cdot & \cdot & \cdot & & \cdot \\
\cdot & \cdot & \cdot & & \cdot \\
A\left(-z_{n}\right) & Q_{s}\left(z_{n}\right) & Q_{s+1}\left(z_{n}\right) & \ldots & Q_{s+n-1}\left(z_{n}\right)
\end{array}\right|
$$

\footnotetext{
1 For convenience in later sections we shall assume that $a_{0}=1$,
} 
with (i)

$$
\begin{gathered}
(A, B)=\left|\begin{array}{cccc}
1 & 1 & \ldots & 1 \\
z_{1} & z_{2} & \ldots & z_{n} \\
\cdot & \cdot & & \cdot \\
z_{1}^{n-2} & z_{2}^{n-2} & \ldots & z_{n}^{n-2} \\
\beta_{1} & \beta_{2} & \ldots & \beta_{n}
\end{array}\right| \div \Delta, \\
\beta_{s}=B\left(-z_{8}\right) \int_{0}^{\infty} \frac{A(x)-A\left(-z_{8}\right)}{x+z_{s}} d \psi(x), \quad s=1,2, \ldots, n,
\end{gathered}
$$

and (ii) $W_{s}(B)$ similar to $(A, B)$ except that the last row of the numerator determinant is replaced by $\gamma_{18}, \gamma_{2 s}, \ldots, \gamma_{n s}$, where $\gamma_{r s}=P_{s}\left(z_{r}\right) B\left(-z_{r}\right)$.

The elements of the determinants in (6a) and (6b) may be irrational or rational, depending upon whether the roots of the polynomial are complex or real. We shall now show how they may be transformed into rational forms, a step making them more amenable for computational purposes.

\section{Rational Forms.}

Let $\Delta U_{s}^{(\lambda)}$ be the product of $\Delta$ and the determinant formed from the array

$$
\left[\begin{array}{ccccc}
z_{1}{ }^{0} & z_{2}{ }^{0} & z_{3}{ }^{0} & \ldots & z_{n}{ }^{0} \\
z_{1}{ }^{1} & z_{2}{ }^{1} & z_{3}{ }^{1} & \ldots & z_{n}{ }^{1} \\
\cdot & \cdot & \cdot & & \cdot \\
z_{t}^{n-1} & z_{2}^{n-1} & z_{3}^{n-1} & \ldots & z_{n}^{n-1} \\
Q_{s}\left(z_{1}\right) & Q_{s}\left(z_{2}\right) & Q_{s}\left(z_{3}\right) & \ldots & Q_{s}\left(z_{n}\right)
\end{array}\right]
$$

after deleting the row with index $\lambda, \lambda=0,1, \ldots, n-1$, and let

$$
\Delta U_{s}^{(\lambda)}=\sum_{r=1}^{n} A_{r}^{(\lambda)} Q_{s}\left(x_{r}\right) \text {. }
$$

Moreover let $\mathbf{A}$ be an $n \times n$ matrix whose $(i, j)$ th element is $A_{j}^{(n-i)}$; similarly let the elements of the $n \times n$ matrices $\mathrm{U}_{s, n}$ and $\mathbf{Q}_{s, n}$ be $U_{s+j-1}^{(n-i)}$ and $Q_{s+j-1}\left(z_{i}\right)$ respectively. Then evidently

$$
\mathrm{AQ}_{s, n}=\Delta \mathrm{U}_{s, n} .
$$

But by postmultiplying $\mathbf{A}$ by the $n \times n$ alternant matrix $\left[z_{i}^{j-1}\right]$ it is found that

$$
|\mathbf{A}|=\Delta^{n-1}
$$


so that

$$
Q_{s}\left(z_{\lambda}\right)_{1}^{n}=\left|U_{s}^{(n-1)} U_{s+1}^{(n-2)}, \ldots, U_{s+n-1}^{(0)}\right| .
$$

Similarly, by premultiplying the bordered matrix of $\mathbf{Q}_{s, n}$ [the determinant of which appears in $(6 \mathrm{~b})]$ by $^{1}$

$$
\left[\begin{array}{cc}
1 & \mathbf{O} \\
\mathbf{O}^{\prime} & \mathbf{A}
\end{array}\right] \text { where } \mathbf{O} \equiv[0,0, \ldots, 0],
$$

it will be found that

$$
P_{s}(A, B)=(-1)^{n}\left|\begin{array}{ccccc}
-(A, B) & W_{s}(B) & W_{s+1}(B) & \ldots & W_{s+n-1}(B) \\
U^{(n-1)}(A) & U_{s}^{(n-1)} & U_{s+1}^{(n-1)} & \ldots & U_{s+n-1}^{(n-1)} \\
\cdot & \cdot & \cdot & & \cdot \\
U^{(0)}(A) & U_{s}^{(0)} & U_{s+1}^{(0)} & \ldots & U_{s+n-1}^{(0)}
\end{array}\right|,
$$

where $U^{(\lambda)}(A)$ is defined by the scheme in (7) except that the last row in the array is replaced by $A\left(-z_{1}\right), A\left(-z_{2}\right), \ldots, A\left(-z_{n}\right)$. The expressions (12) and (11) for the numerator and denominator of the generalised C.F. associated with $F\left(z_{\lambda}\right)_{1}^{n}$ are now in terms of rational elements, each element being expressible in terms of the symmetric functions of the roots of $C(x)=0$.

It is to be noted that we may write

$$
W_{s}(B)=\sum_{r=1}^{n}(-1)^{n-r} b_{r} V_{s}^{(n-r)}
$$

where $B(x)=\sum_{r=1}^{n} b_{r} x^{n-r}$ and $^{2} V_{s}^{(\lambda)}, \lambda=0,1, \ldots,(n-1)$, is similar to $U_{s}^{(n-1)}$ except that the last row of the numerator determinant is

$$
z_{1}^{\lambda} P_{s}\left(z_{1}\right), z_{2}^{\lambda} P_{s}\left(z_{2}\right), \ldots, z_{n}^{\lambda} P_{s}\left(z_{n}\right) .
$$

If the degree of $A(x)$ is less than $n$, the rôles of $A(x)$ and $B(x)$ may be interchanged, yielding the identity

$$
P_{s}(A, B)=P_{s}(B, A) \text {. }
$$

Again, if $A(x) B(x)=A^{*}(x) B^{*}(x)$, where $A^{*}(x)$ and $B^{*}(x)$ are poly-

a In this matrix $\boldsymbol{O}^{\prime}$ means a column matrix of $n$ components.

2 It will be observed that $V^{(\lambda)}$ is the $n$th divided difference of $z^{\lambda} P_{d}(z)$ with respect to the arguments $z_{1}, z_{2}, \ldots, z_{n}$. Similarly $U_{g^{(n-1)}}$ is the $n$th divided difference of $Q_{n}(z)$. 
nomials, the degree of $B^{*}(x)$ being less than $n$, then

$$
\begin{aligned}
F\left(z_{\lambda}\right)_{1}^{n} & =\lim _{s \rightarrow \infty} \frac{P_{s}\left(A^{*}, B^{*}\right)}{Q_{s}\left(z_{\lambda}\right)_{1}^{n}} \\
& =\operatorname{li.is.~}_{s \rightarrow \infty} \frac{P_{s}\left(B^{*}, B^{*}\right)}{Q_{s}\left(z_{\lambda}\right)_{1}^{n}} \text { if } A^{*}=B^{*} .
\end{aligned}
$$

The only modification required in (11) and (12) when the roots $z_{\lambda}$ are not distinct is to replace each alternant by its confluent form; this presents no difficulties since each element is the ratio of alternants in the roots $z_{\lambda}$.

4. Recurrence relation satisfied by $V_{s}^{(\lambda)}, U_{s}^{(\lambda)}$.

It will be seen that $V_{s}^{(\lambda)}$ and $U_{s}^{(\lambda)}$ are linear functions of $P_{s}\left(z_{j}\right)$ and $Q_{s}\left(z_{j}\right), j=1,2, \ldots, n$, respectively, the coefficients being independent of $s$. We shall now prove that they satisfy the same recurrence relation, and that this recurrence relation can be directly derived from that satisfied by $P_{s}(x)$ or $Q_{s}(x)$. We require the following theorem.

Lемma. If the polynomial $T_{s}(x)$ is defined by

$$
\begin{gathered}
T_{s+1}(x)=\left(x+c_{s+1}\right) T_{s}(x)-\tilde{a}_{s} T_{s-1}(x), \quad s=0,1, \ldots, \\
T_{0}(x)=1, \quad \tilde{a}_{s}=a_{s} \text { for } s>0, \quad \tilde{a}_{s}=0 \text { for } s \leqslant 0
\end{gathered}
$$

then 1

$$
\prod_{\lambda=1}^{n}\left(x-z_{\lambda}\right) T_{s}(x)=\prod_{\lambda=1}^{n}\left(\zeta_{s}-z_{\lambda}\right) T_{s}(x), \quad s=0,1, \ldots,
$$

where $\zeta_{s}$ is the finite difference operator $\mathbf{E}-c_{s+1}+\tilde{a}_{s} \mathbf{E}^{-1}$ and $\mathbf{E} T_{s}(x)=T_{s+1}(x)$.

Proof. This proceeds on inductional lines. For $n=1$

$$
\begin{aligned}
\left(x-z_{1}\right) T_{s}(x) & =T_{s+1}(x)-\left(z_{1}+c_{s+1}\right) T_{s}(x)+\tilde{a}_{s} T_{s-1}(x), \\
& =\left(\zeta_{s}-z_{1}\right) T_{s}(x), \quad s=0,1,2, \ldots,
\end{aligned}
$$

and for $n=2$

$$
\begin{aligned}
& \left(x-z_{1}\right)\left(x-z_{2}\right) T_{s}(x) \\
& =\left(x-z_{2}\right) T_{s+1}(x)-\left(x-z_{2}\right)\left(z_{1}+c_{s+1}\right) T_{s}(x)+\tilde{a}_{s}\left(x-z_{2}\right) T_{s-1}(x), \quad s=0,1, \ldots, \\
& =\left(\zeta_{s+1}-z_{2}\right) T_{s+1}(x)-\left(z_{1}+c_{s+1}\right)\left(\zeta_{s}-z_{2}\right) T_{s}(x)+\tilde{a}_{s}\left(\zeta_{s-1}-z_{2}\right) T_{s-1}(x), \\
& \qquad \cdot \quad s=1,2, \ldots, \\
& =\left\{\mathbf{E}\left(\zeta_{s}-z_{2}\right)-\left(z_{1}+c_{s+1}\right)\left(\zeta_{s}-z_{2}\right)+\tilde{a}_{s} \mathbf{E}^{-1}\left(\zeta_{s}-z_{2}\right)\right\} T_{s}(x) \\
& =\left(\zeta_{s}-z_{1}\right)\left(\zeta_{s}-z_{2}\right) T_{s}(x) \text { for } s>0 .
\end{aligned}
$$

1 In evaluating this expression a particular value of $s$ can only be inserted after the operational symbols have been absorbed. 
It may also be shown to hold for $s=0$, when the fact that $\tilde{a}_{0}=\tilde{a}_{-1}=0$ conserves the identity. Now assume the truth of the statement for polynomials $\Pi\left(x-z_{\lambda}\right)$ of degree less than or equal to $n-1$. Then

$$
\begin{aligned}
& \prod_{\lambda=1}^{n}\left(x-z_{\lambda}\right) T_{s}(x)=\prod_{\lambda=1}^{n-1}\left(x-z_{\lambda}\right)\left\{T_{s+1}(x)-\left(z_{n}+c_{s+1}\right) T_{s}(x)+\tilde{a}_{s} T_{s-1}(x)\right\}, \quad s \geqslant 0, \\
& =\prod_{\lambda=1}^{n-1}\left(\zeta_{s+1}-z_{\lambda}\right) T_{s+1}(x)-\left(z_{n}+c_{s+1}\right) \prod_{\lambda=1}^{n-1}\left(\zeta_{s}-z_{\lambda}\right) T_{s}(x) \\
& +\tilde{a}_{\delta_{\lambda=1}}^{n-1}\left(\zeta_{s-1}-z_{\lambda}\right) T_{s-1}(x) \text { for } s \geqslant 1
\end{aligned}
$$

Now, if $s=0$,

$$
\begin{aligned}
\prod_{\lambda=1}^{n}\left(x-z_{\lambda}\right) T_{0}(x) & =\prod_{\lambda=1}^{n-1}\left(x-z_{\lambda}\right)\left\{T_{1}(x)-\left(z_{n}+c_{1}\right) T_{0}(x)\right\} \\
& =\prod_{\lambda=1}^{n-1}\left(x-z_{\lambda}\right)\left\{T_{1}(x)-\left(z_{n}+c_{1}\right) T_{0}(x)+\tilde{a}_{0} T_{-1}(x)\right\} .
\end{aligned}
$$

Thus (16) still holds for $s=0$. Hence

$$
\prod_{\lambda=1}^{n}\left(x-z_{\lambda}\right) T_{s}(x)=\left(\mathbf{E}-\left(z_{n}+c_{s+1}\right)+\tilde{a}_{s} \mathbf{E}^{-1}\right) \prod_{\lambda=1}^{n-1}\left(\zeta_{s}-z_{\lambda}\right) T_{s}(x), \quad s \geqslant 0,
$$

from which the truth of the lemma follows. It is now evident that

$$
\prod_{\lambda=1}^{n}\left(\zeta_{s}-z_{\lambda}\right) T_{s}(x)=0 \text { for }\left\{\begin{array}{l}
x=z_{1}, z_{2}, \ldots, z_{n} \\
s \geqslant 0
\end{array}\right.
$$

and indeed if

$$
\Omega_{s}=\sum_{r=1}^{n} q_{r} T_{s}\left(z_{r}\right)
$$

then

$$
\prod_{\lambda=1}^{n}\left(\zeta_{s}-z_{\lambda}\right) \Omega_{s}=0, s \geqslant 0,
$$

which is the recurrence relation for $\Omega_{s}$. (It is to be understood that $q_{r}$ is independent of $s$.)

We now apply the lemma and (18) to the polynomials appearing in the numerators and denominators of C.F. (4); i.e. to $P_{s}(z)$ and $Q_{s}(z)$, where

$$
\text { - } \frac{P_{s}(z)}{Q_{s}(z)}=\frac{a_{0}}{z+c_{1}}-\frac{a_{1}}{z+c_{2}}-\frac{a_{2}}{z+c_{3}}-\ldots \quad-\frac{a_{s-1}}{z+c_{s}}\left\{\begin{array}{l}
s=1,2, \ldots \\
a_{0}=1
\end{array}\right.
$$

with

$$
\left\{\begin{array}{l}
Q_{0}=1, \quad Q_{1}=z+c_{1}, \\
P_{0}=0, \quad P_{1}=1
\end{array}\right.
$$


It is evident that we have proved that the recurrence relation followed by $U_{s}^{(\lambda)}$ defined in (8) is

$$
\left\{\begin{array}{l}
\begin{array}{l}
C\left(-\zeta_{s}\right) U_{s}^{(\lambda)}=0 \\
C\left(-\mathbf{E}+c_{s+1}-\tilde{a}_{s} \mathbf{E}^{-1}\right) U_{s}^{(\lambda)}=0 \\
s=0,1,2, \ldots ; \lambda=0,1, \ldots, n-1
\end{array}
\end{array}\right.
$$

where $\tilde{a}_{s}=a_{s}$ for $s>0, \tilde{a}_{s}=0$ for $s \leqslant 0$, the $a$ 's and $c$ 's being given in (19). Similarly after a slight modification, the recurrence followed by $V_{s}^{(\lambda)}$ is found to be

$$
C\left(-\mathbf{E}+c_{s+1}-\hat{a}_{s} \mathbf{E}_{s}^{-1}\right) V_{s}^{(\lambda)}=0, \quad s=1,2, \ldots,
$$

where $\hat{a}_{s}=a_{s}$ for $s>1, \hat{a}_{s}=0$ for $s \leqslant 1$.

\section{A fundamental. system of solutions.}

The recurrence relations (20a) and (20b), each of order $2 n$, are exactly the same for $s \geqslant 1$. We shall now prove that $U_{s}^{(\lambda)}, V_{s}^{(\lambda)}, \lambda=0,1,2, \ldots, n-1$, is a fundamental system of solutions with respect to this common recurrence equation. For consider

$$
\Phi_{s, n}=\left[\begin{array}{cc}
\mathbf{U}_{s, n} & \mathbf{U}_{s+n, n} \\
\mathbf{J V}_{s, n} & \mathbf{J V}_{s+n, n}
\end{array}\right],
$$

where $\mathbf{V}_{s, n}$ is the same matrix function of the V's as $\mathbf{U}_{s, n}$ is of the $U^{\prime} s$, and $\mathbf{J}$ is an $n \times n$ matrix with zeros everywhere except in the secondary diagonal, where there are units. It will be seen that

$$
\left[\begin{array}{cc}
\mathbf{A} & \mathbf{0} \\
\mathbf{O}^{\prime} & \mathbf{A}^{*}
\end{array}\right]\left[\begin{array}{ll}
\mathbf{Q}_{s, n} & \mathbf{Q}_{s+n, n} \\
\mathbf{P}_{s, n} & \mathbf{P}_{s+n, n}
\end{array}\right]=\Delta \boldsymbol{\Phi}_{s, n}
$$

where $\mathbf{P}_{s, n}$ is a matrix similar to $\mathbf{Q}_{s, n}$, with $P(z)$ replacing $Q(z)$, and

$$
\mathbf{A}^{*}=\left[\begin{array}{cccc}
1 & 1 & \ldots & 1 \\
z_{1} & z_{1} & \ldots & z_{n} \\
\cdot & \cdot & \ldots & \cdot \\
z_{1}^{n-1} & z_{2}^{n-1} & \ldots & z_{n}^{n-1}
\end{array}\right]\left[\begin{array}{cccccc}
A_{1}^{(n-1)} & 0 & . & . & . & 0 \\
0 & A_{2}^{(n-1)} & 0 & . & . & 0 \\
0 & 0 & . & . & . & A_{n}^{(n-1)}
\end{array}\right]
$$

Hence, taking determinants in (22), we have

$$
\Delta^{2 n}\left|\Phi_{s n}\right|=|\mathbf{A}| \cdot\left|\mathbf{A}^{*}\right|(-1)^{\frac{1}{2} n(n-1)} \Theta_{s}\left(z_{1}, z_{2}, \ldots, z_{n}\right),
$$


where

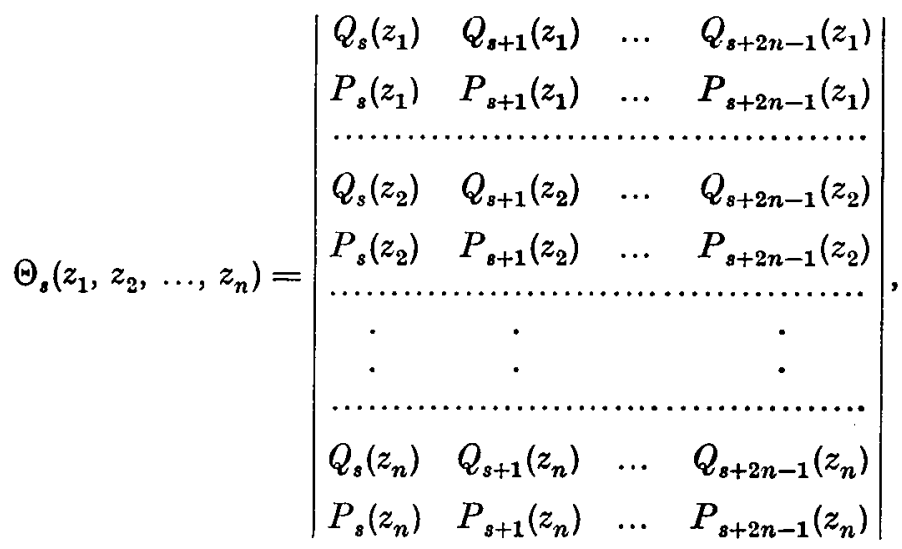

the general expression of this type being $\Theta_{s+r}\left(z_{r+1}, z_{r+2}, \ldots, z_{n}\right)$, in which $s$ indicates the suffix in the first column of the determinant, which is of order $2(n-r)$, the arguments $z_{r+1}, z_{r+1}, z_{r+2}, z_{r+2}$, etc. occurring in successive rows.

Now $P_{s}(x)$ and $Q_{s}(x)$ follow

$$
y_{s}=\left(x+c_{s}\right) y_{s-1}-a_{s-1} y_{s-2}
$$

Using this on the last column of (24) and eliminating $Q_{s+2 n-1}\left(z_{1}\right)$ and $P_{s+2 n-1}\left(z_{1}\right)$, and so on until all the elements in the first two rows except the first four are removed, we find

$$
\Theta_{s}\left(z_{1}, z_{2}, \ldots, z_{n}\right)=\alpha_{s} \Theta_{s+1}\left(z_{2}, z_{3}, \ldots, z_{n}\right) \prod_{\lambda=2}^{n}\left(z_{\lambda}-z_{1}\right)^{2},
$$

where $\alpha_{s}=\prod_{\lambda=0}^{s} a_{\lambda}=\left|Q_{s}(x), P_{s+1}(x)\right|$.

Continuing the condensation process in evidence in (25), it will be found that

$$
\begin{aligned}
\Theta_{s}\left(z_{1}, z_{2}, \ldots, z_{n}\right) & =\alpha_{8} \prod_{2}^{n}\left(z_{\lambda}-z_{1}\right)^{2} \cdot \alpha_{s+1} \prod_{3}^{n}\left(z_{\lambda}-z_{2}\right)^{2} \ldots \alpha_{8+n-2}\left(z_{n}-z_{n-1}\right)^{2} \cdot \alpha_{s+n-1} \\
& =\Delta^{2} \prod_{v=8}^{s+n-1} \prod_{\lambda=0}^{v} a_{\lambda} .
\end{aligned}
$$

But $\left|\mathbf{A}^{*}\right|=(-1)^{\frac{1}{2 n(n-1)}} \Delta^{n-1}$ and from (10) $|\mathbf{A}|=\Delta^{n-1}$, so that from (23)

$$
\left|\Phi_{s, n}\right|=\prod_{v=s}^{s+n-1} \prod_{\lambda=0}^{v} a_{\lambda} \text {. }
$$


We have thus proved that $U_{s}^{(\lambda)}, V_{s}^{(\lambda)}, \lambda=0,1,2, \ldots, n-1$ form a fundamental system of solutions of the recurrence relation (20). It is evident that the result still holds when $C(x)=0$ has multiple roots.

\section{Illustration.}

We consider $n=3$ and $C(x)=(x+\alpha)(x+\beta)(x+\gamma)>0$ for $t \geqslant 0$, and the integral

$$
I_{m}=\int_{0}^{\infty} \frac{x^{m} d \psi(x)}{(x+\alpha)(x+\beta)(x+\gamma)}, \quad m=0,1,2,
$$

where it is assumed $\psi(x)$ satisfies the conditions of $\S 2$. The denominators of the associated generalised 3rd order C.F. all have the form

$$
Q_{s}(\alpha, \beta, \gamma)=\left|U_{s}^{(2)}, U_{s+1}^{(1)}, U_{s+2}^{(0)}\right|
$$

where

$$
\begin{aligned}
& U_{s}^{(2)}=\left|\begin{array}{ccc}
1 & 1 & 1 \\
\alpha & \beta & \gamma \\
Q_{s}(\alpha) & Q_{s}(\beta) & Q_{s}(\gamma)
\end{array}\right| \div\left|\alpha^{0}, \beta^{1}, \gamma^{2}\right|, \\
& U_{s}^{(1)}=\left|\begin{array}{ccc}
1 & 1 & 1 \\
\alpha^{2} & \beta^{2} & \gamma^{2} \\
Q_{s}(\alpha) & Q_{s}(\beta) & Q_{s}(\gamma)
\end{array}\right| \div\left|\alpha^{0}, \beta^{1}, \gamma^{2}\right|, \\
& U_{s}^{(0)}=\left|\begin{array}{ccc}
\alpha & \beta & \gamma \\
\alpha^{2} & \beta^{2} & \gamma^{2} \\
Q_{s}(\alpha) & Q_{s}(\beta) & Q_{s}(\gamma)
\end{array}\right| \div\left|\alpha^{0}, \beta^{1}, \gamma^{2}\right| .
\end{aligned}
$$

The confluent forms are easily constructed ${ }^{1}$; for example ${ }^{2}$ if

(i) $\beta=\gamma$

$$
U_{s}^{(2)}=\left|\begin{array}{ccc}
1 & 1 & 0 \\
\alpha & \beta & 1 \\
Q_{s}(\alpha) & Q_{s}(\beta) & Q_{s}^{\prime}(\beta)
\end{array}\right| \div\left|\begin{array}{ccc}
1 & 1 & 0 \\
\alpha & \alpha & 1 \\
\alpha^{2} & \beta^{2} & 2 \beta
\end{array}\right|,
$$

(ii) $\alpha=\beta=\gamma$

$$
U_{s}^{(2)}=\left|\begin{array}{ccc}
1 & 0 & 0 \\
\alpha & 1 & 0 \\
Q_{s}(\alpha) & Q_{s}(\alpha) & \frac{1}{2} Q_{s}^{\prime}(\alpha)
\end{array}\right| \div\left|\begin{array}{ccc}
1 & 0 & 0 \\
\alpha & \beta & 0 \\
\alpha^{2} & 2 \alpha & 1
\end{array}\right| .
$$

1 See for example H. W. Turnbull, Theory of Equations, p. 48 (Edinburgh, 1946).

Primes indicate derivatives. 
For the numerators there are several possibilities, which we briefly indicate. Let $A_{s}=x^{s}, s=0,1,2$; then it is easily seen that $U^{(r)}\left(A_{s}\right)=\delta_{r, s}, r=0,1,2$ where $\delta$ is the Kronecker delta symbol. Moreover,

$$
\begin{aligned}
W_{s}\left(A_{\lambda}\right) & =(-1)^{\lambda}\left|\begin{array}{ccc}
1 & 1 & 1 \\
\alpha & \beta & \gamma \\
\alpha^{\lambda} P_{s}(\alpha) & \beta^{\lambda} P_{s}(\beta) & \gamma^{\lambda} P_{s}(\gamma)
\end{array}\right| \div\left|\alpha^{0}, \beta^{1}, \gamma^{2}\right| \\
& =(-1)^{\lambda} V_{s}^{(\lambda)},
\end{aligned}
$$

and from (6c)

$$
\left(A_{r}, A_{s}\right)=0, \quad r+s \leqslant 2, \quad r, s=0,1,2 .
$$

We now have

$$
\begin{gathered}
I_{0}=\operatorname{li.s.s.~}_{s \rightarrow \infty} \frac{P_{s}\left(A_{0}, A_{0}\right)}{Q_{s}}, \\
I_{1}=\lim _{s \rightarrow \infty} \frac{P_{s}\left(A_{1}, A_{0}\right)}{Q_{s}}=\lim _{s \rightarrow \infty} \frac{P_{s}\left(A_{0}, A_{1}\right)}{Q_{s}}, \\
I_{2}=\lim _{s \rightarrow \infty} \frac{P_{s}\left(A_{2}, A_{0}\right)}{Q_{s}}=\lim _{s \rightarrow \infty} \frac{P_{s}\left(A_{0}, A_{2}\right)}{Q_{s}}=\underset{s \rightarrow \infty}{\text { li.s. }} \frac{P_{s}\left(A_{1}, A_{1}\right)}{Q_{s}},
\end{gathered}
$$

where $Q_{s} \equiv Q_{s}(\alpha, \beta, \gamma)$ and

$$
\begin{aligned}
& P_{s}\left(A_{0}, A_{0}\right)=\left|\begin{array}{lll}
V_{8}^{(0)} & V_{8+1}^{(0)} & V_{8+2}^{(0)} \\
U_{8}^{(2)} & U_{8+1}^{(2)} & U_{8+2}^{(2)} \\
U_{8}^{(1)} & U_{8+1}^{(1)} & U_{8+2}^{(1)}
\end{array}\right| \\
& P_{s}\left(A_{1}, A_{0}\right)=-\left|\begin{array}{lll}
V_{8}^{(0)} & V_{8+1}^{(0)} & V_{s+2}^{(0)} \\
U_{8}^{(2)} & U_{8+1}^{(2)} & U_{s+2}^{(2)} \\
U_{8}^{(0)} & U_{8+1}^{(0)} & U_{s+2}^{(0)}
\end{array}\right|=-\left|\begin{array}{lll}
V_{8}^{(1)} & V_{8+1}^{(1)} & V_{8+2}^{(1)} \\
U_{8}^{(2)} & U_{s+1}^{(2)} & U_{8+2}^{(2)} \\
U_{s}^{(1)} & U_{s+1}^{(1)} & U_{8+2}^{(1)}
\end{array}\right|=P_{s}\left(A_{0}, A_{1}\right), \\
& P_{s}\left(A_{2}, A_{0}\right)=\left|\begin{array}{lll}
V_{s}^{(0)} & V_{s+1}^{(0)} & V_{s+2}^{(0)} \\
U_{s}^{(1)} & U_{s+1}^{(1)} & U_{s+2}^{(1)} \\
U_{s}^{(0)} & U_{s+1}^{(0)} & U_{s+2}^{(0)}
\end{array}\right|=\left|\begin{array}{ccc}
V_{s}^{(2)} & V_{s+1}^{(2)} & V_{s+2}^{(2)} \\
U_{s}^{(2)} & U_{s+1}^{(2)} & U_{s+2}^{(2)} \\
U_{s}^{(1)} & U_{s+1}^{(1)} & U_{s+2}^{(1)}
\end{array}\right|=P_{s}\left(A_{0}, A_{2}\right), \\
& P_{8}\left(A_{1}, A_{1}\right)=\left|\begin{array}{ccc}
V_{8}^{(1)} & V_{8+1}^{(1)} & V_{s+2}^{(1)} \\
U_{8}^{(2)} & U_{8+1}^{(2)} & U_{8+2}^{(2)} \\
U_{8}^{(0)} & U_{8+1}^{(0)} & U_{8+2}^{(0)}
\end{array}\right|
\end{aligned}
$$


Recurrence relation for $V_{s}^{(\lambda)}$ and $U_{s}^{(\lambda)}$. From (20a) and (20b) this is

$$
\begin{gathered}
\left(\mathbf{E}-c_{s+1}+\tilde{a}_{s} \mathbf{E}^{-1}-\alpha\right)\left(\mathbf{E}-c_{s+1}+\tilde{a}_{s} \mathbf{E}^{-1}-\beta\right)\left(\mathbf{E}-c_{s+1}+\tilde{a}_{s} \mathbf{E}^{-1}-\gamma\right) U_{s}^{(\lambda)}=0, \\
s \geqslant 0,
\end{gathered}
$$

with a similar expression for $V_{8}^{(\lambda)}$. On expansion this becomes

$$
\begin{aligned}
U_{s+3}^{(\lambda)}-\lambda_{s} U_{s+2}^{(\lambda)}+\mu_{s} U_{s+1}^{(\lambda)} & -\nu_{s} U_{s}^{(\lambda)}+\tilde{a}_{s} \mu_{s-1} U_{s-1}^{(\lambda)}-\tilde{a}_{s} \tilde{a}_{s-1} \lambda_{s-2} U_{s-2}^{(\lambda)} \\
& +\tilde{a}_{s} \tilde{a}_{s-1} \tilde{a}_{s-2} U_{s-3}^{(\lambda)}=0, \quad \lambda=0,1,2 ; \quad s \geqslant 0,
\end{aligned}
$$

where

$$
\left\{\begin{array}{c}
\lambda_{s}=\alpha+\beta+\gamma+c_{s+1}+c_{s+2}+c_{s+3} \\
\mu_{s}=\beta \gamma+\gamma \alpha+\alpha \beta+(\alpha+\beta+\gamma)\left(c_{s+1}+c_{s+2}\right)+c_{+1}^{2}+c_{s+1} c_{s+2}+c_{s+2}^{2} \\
\quad+\tilde{a}_{s}+\tilde{a}_{s+1}+\tilde{a}_{s+2} \\
v_{s}=\left(\alpha+c_{s+1}\right)\left(\beta+c_{s+1}\right)\left(\gamma+c_{s+1}\right)+(\alpha+\beta+\gamma)\left(\tilde{a}_{s}+\tilde{a}_{s+1}\right) \\
\quad+\tilde{a}_{s} c_{s}+2 \tilde{a}_{s} c_{s+1}+2 \tilde{a}_{s+1} c_{s+1}+\tilde{a}_{s+1} c_{s+2},
\end{array}\right.
$$

and

$$
\left\{\begin{array}{lll}
U_{0}^{(0)}=1 & U_{1}^{(0)}=c_{1} & U_{2}^{(0)}=c_{1} c_{2}-a_{1} \\
U_{0}^{(1)}=0 & U_{1}^{(1)}=-1 & U_{2}^{(1)}=-c_{1}-c_{2} \\
U_{0}^{(2)}=0 & U_{1}^{(2)}=0 & U_{2}^{(2)}=1
\end{array}\right.
$$

with $\tilde{a}_{s}=a_{s}, s>0 ; \tilde{a}_{s}=0, s \leqslant 0 ;$ and $a_{s}, c_{s}$ are given in the C.F. in (4). Similarly $V_{s}^{(\lambda)}$ follows (33) for $s \geqslant 1$ provided $\tilde{a}_{s}$ is replaced throughout by $\hat{a}_{s}$ where $\hat{a}_{s}=a_{s}, s>1 ; \hat{a}_{s}=0, s \leqslant 1$; and the initial values are

$$
\left\{\begin{array}{llll}
V_{0}^{(0)}=0 & V_{1}^{(0)}=0 & V_{2}^{(0)}=0 & V_{3}^{(0)}=1 \\
V_{0}^{(1)}=0 & V_{1}^{(1)}=0 & V_{2}^{(1)}=1 & V_{3}^{(1)}=\alpha+\beta+\gamma+c_{2}+c_{3} \\
V_{0}^{(2)}=0 & V_{1}^{(2)}=1 & V_{2}^{(2)}=\alpha+\beta+\gamma+c_{2} & V_{3}^{(2)}=\alpha^{2}+\beta^{2}+\gamma^{2}+\beta \gamma+\gamma \alpha+\alpha \beta \\
& & & +\left(c_{2}+c_{3}\right)(\alpha+\beta+\gamma) \\
& & & +c_{2} c_{3}-a_{2} .
\end{array}\right.
$$

The recurrence relation (33) is the same (apart from minor changes due to the notation used) as that given for $U_{s}, V_{s}, W_{s}, X_{s}$ under expression (13) in [5]. With the present notation and approach, however, there is no difficulty in writing down the recurrence relation for $V_{s}^{(\lambda)}$ and $U_{s}^{(\lambda)}$ together with their initial values. 
7. Remarks on generalised C.F.'s.

Previous attempts at generalising C.F.'s have to a certain extent consisted in extending the recurrence relation satisfied by the numerator and denominator, contributions having been made by Jacobi [6], Hermite [7], Perron [8], Bateman [9], and Paley and Ursell [10]. In addition it seems that Stieltjes was very close to the theory developed here, for in the correspondence between Stieltjes and Hermite [11] Stieltjes indicated (see letter 167 written in 1889) that he had perfected a method of approximating the integral

$$
P=\int_{a}^{b} \frac{f(x)}{\phi(x)} d x
$$

using least squares. In fact he minimises the expression

$$
R_{n}=\int_{a}^{b} \frac{f(x)}{\phi(x)}\left\{1+x_{1} \phi+x_{2} \phi^{2}+\ldots+x_{n} \phi^{n}\right\}^{2} d x
$$

with respect to $x_{1}, x_{2}, \ldots, x_{n}$ and obtains the approximation

where

$$
P=-\left|\begin{array}{ccccc}
0 & c_{0} & c_{1} & \ldots & c_{n-1} \\
c_{0} & c_{1} & c_{2} & \ldots & c_{n} \\
\cdot & \cdot & \cdot & \ldots & \cdot \\
c_{n-1} & c_{n} & c_{n+1} & \ldots & c_{2 n-1}
\end{array}\right| \div\left|c_{1}, c_{3}, \ldots, c_{2 n-1}\right|
$$

$$
c_{k}=\int_{a}^{b} \phi^{k} f(x) d x .
$$

Stieltjes goes on to show that there is an alternative form

$$
P=\sum_{1}^{n} \frac{A_{s}^{2}}{B_{s-1} B_{s}} \quad \text { where } \quad\left\{\begin{array}{l}
A_{s}=\left|c_{0}, c_{2}, \ldots, c_{2 s-2}\right| \\
B_{s}=\left|c_{1}, c_{3}, \ldots, c_{2 s-1}\right|
\end{array}\right.
$$

and then disposes of the remainder when the limits of integration are finite $^{1}$. As an application he derives the even part of the C.F. for

$$
\int_{a}^{b} \frac{f(x) d x}{z-x} \text {. }
$$

1 As far as I can gather Stieltjes does not state the restrictions on $\phi$ and $f$, but his method of proving that the remainder vanishes in the limit is noteworthy. Apparently, another letter by Stieltjes on this topic has been lost according to a footnote by the editors. 
Hermite (letter 168 in the correspondence) remarks " votre nouveau point de vue pour obtenir l'approximation de l'intégrale

$$
\int_{a}^{b} \frac{f(x) d x}{z-x}
$$

constitue un très heureux et très grand progrès . ..'. However, as far as I can trace the only later references to this development [see Stieltjes (1889), "Sur un développement en fraction continue", Comptes Rendus cviii, p. 1297; also Stieltjes (1918) Oeuvres Complètes, "Recherches sur les fractions continues", Ch. viii, pp. 500-502. Groningen] suggest that the broader possibilities were not envisaged. It is perhaps worth recording here that the generalisation we have developed, springs from the minimisation of the expression

$$
\int_{a}^{b} C(x)\left\{\frac{A(x)}{C(x)}-\pi_{n}(x)\right\}^{2} d \psi(x)
$$

with respect to the coefficients in the polynomial $\pi_{n}, C(x)$ being in general positive in $(a, b)$, convergence questions being settled by invoking Parseval's theorem. The difficulties in generalising (34) by taking $\phi$ to be a quadratic or polynomial of higher degree are evident.

Lastly we may remark that Hermite evidently ${ }^{1}$ gave some thought to the question of generalising C.F's, but his contribution to the subject in 1893 [7] appears to have no relation to (33) or (34).

\section{REFERENCES.}

1. L. R. Shenton, Proc. Edinburgh Math. Soc. (2), 9 (1953), 44-52.

2. ——, ibid. (2), 10 (1954), 78-91.

3. ——, ibid. (2), 10 (1956), 134-140.

4. —, ibid. (2), 10 (1957), 153-166.

5. ——, ibid. (2), 10 (1957), 167-188.

6. C. G. J. Jacobi, Crelle's Journal, lxix (1868), 29-64.

7. Ch. Hermite, Annali di Mat. (2), 21 (1893), 289-308.

8. O. Perron, Math. Annalen, 64 (1907), 1-76.

9. H. Bateman, Quart. J. Math., xli (1910), 302-308.

10. R. E. A. C. Paley and H. D. Ursell, Proc. Cambridge Phil. Soc., 26 (1929), 127-144.

11. Stieltjes and Hermite, Correspondance d'Hermite et de Stieltjes, Vols. 1 and 2 (Paris, 1905: Gauthier-Villars).

\section{Manchester College of Science and Technology, MaNCheSter, 1 .}

1 There are about a dozen references to the subject in letters written by Hermite to Stieltjes; see [11], Tome 2 and, for example, letters 234-236, 241, 242, 245, 265, 278, 388, 389,391 and 392 . 